\title{
Die Stärken nutzen: Vorschläge zur Weiterentwicklung des Gesundheitsfonds
}

Allen Skeptikern zum Trotz, jetzt ist er tatsächlich in Kraft: Zum 1. Januar 2009 wurde der letzte Teil der Gesundheitsreform der Großen Koalition wirksam, der viel gescholtene Gesundheitsfonds wurde eingeführt. Selten waren sich Vertreterinnen und Vertreter aus Politik und Wissenschaft so einig in ihrer Kritik an einer Gesundheitsreform, deren Ergebnis von der Notwendigkeit zum politischen Kompromiss zwischen sehr unterschiedlichen Vorstellungen zur Finanzierung des Gesundheitswesens geprägt war. Gleichzeitig liegt zumindest darin ein gewisser Charme der Kompromisslösung, dass diese bewusst offen für die Weiterentwicklung (nach geänderten politischen Mehrheitsverhältnissen) in verschiedene Richtungen angelegt wurde. Dieser Diskussionsbeitrag ist auf die Zukunftsperspektiven des Gesundheitssystems gerichtet und nimmt diese Option ernst. Die nun in Kraft getretene Reform ist mit einer Reihe gravierender Probleme verbunden. ${ }^{1}$ Die hier zur Diskussion gestellte These ist dennoch, dass das Rad nicht zum Status quo ante zurückgedreht werden muss, um ein nachhaltiges und solidarisch finanziertes Gesundheitssystem zu entwickeln. Stattdessen sollte an durchaus vorhandenen Stärken des Gesundheitsfonds angeknüpft werden. Dazu ist es allerdings notwenig, vorhandene Fehlkonstruktionen rasch zu beheben und weitere Begleitmaßnahmen einzuführen. Folgende Aspekte sind dafür künftig von besonderer Bedeutung:

\section{ERWEITERUNG DER FINANZIERUNGS- BASIS DURCH STEUERN}

Nicht für alles, was in der öffentlichen Diskussion mit dem Fonds in Verbindung gebracht wurde, ist dieser direkt verantwortlich. So liegen etwa die Wurzeln der Beitragssatzsteigerungen in der Gesetzlichen Krankenversicherung (GKV) nicht im
Fonds selbst, sondern unter anderem in höheren Kosten für Ärztehonorare sowie gestiegenen Personalkosten der Krankenhäuser. Die Fondskonstruktion führte lediglich dazu, dass Beitragssatzsteigerungen zu einem bestimmten Zeitpunkt gebündelt offenbar wurden. Zu Recht wird allerdings kritisiert, dass im Zusammenspiel aller Maßnahmen des GKV-Wettbewerbsstärkungsgesetzes eines der zentralen Ausgangsziele der Reform - die Stabilisierung der Finanzierungsgrundlage und Beitragssätze - nicht gelungen ist. Die strukturelle Einnahmeschwäche der GKV wurde nicht gelöst, da sowohl die Begrenzung der Beitragspflicht auf Löhne und Gehälter als auch der beitragspflichtige Personenkreis im Wesentlichen unverändert blieb. Der Ausbau der Steuerfinanzierung blieb zaghaft, die längerfristige Gegenfinanzierung unklar.

Eine gewisse Trendwende brachte erst das Konjunkturpaket. Wenn dies in Kraft tritt, steigt der Steuerzuschuss rascher als geplant auf $14 \mathrm{Mrd} . €$ an. Eine stärkere Steuerfinanzierung würde das Gesundheitssystem auf eine nachhaltigere Finanzierungsbasis stellen. Denn die Gesundheitsausgaben haben sich in der Vergangenheit ähnlich wie das Bruttoinlandsprodukt entwickelt, doch die Einnahmenbasis der GKV - die Gehälter der sozialversicherungspflichtig Beschäftigten - konnte damit nicht Schritt halten. Dadurch eingeleitete Beitragssatzsenkungen können Wachstumseffekte erzielen und die Steuerzuschüsse würden - zumindest solange die Private Kranken-Vollversicherung in der heutigen Form bestehen bleibt - mehr Gerechtigkeit ins System bringen. Denn über das Steuersystem würden sowohl die heute privat versicherten Berufs- und Einkommensgruppen in das Solidarsystem einbezogen als auch Kapital- und Mieteinkünfte berücksichtigt. Eine solche Lösung ist mit weniger Bürokratie verbunden als der von
Befürwortern einer Bürgerversicherung vertretene Ansatz einer separaten Verbeitragung von Kapitaleinkommen.

\section{VERSICHERUNGSFREMDE LEISTUNGEN}

Längerfristig sollte es das Ziel sein, zumindest die versicherungsfremden Leistungen in der GKV vollständig durch Steuermittel auszugleichen. Dies würde ein Potenzial von über $20 \mathrm{Mrd}$ € beinhalten, wenn man darunter die Leistungen für Schwangerschaft und Mutterschaft sowie das Mutterschaftsgeld (ca. 4 Mrd.€), die Beitragsfreiheit von Kindern (ca. 14 Mrd.€) sowie die reduzierten Beiträge für Empfänger von Arbeitslosengeld II (ca. 4,7 Mrd.€) fasst. ${ }^{2}$

Die Beitragsfreiheit im Rahmen von Ehen stellt einen Sonderfall dar. Auf den ersten Blick läge es nahe, auch die Mitversicherung der Ehepartnerinnen und -partner in die Steuerfinanzierung einzubeziehen. Von der feministischen Wohlfahrtsstaatsforschung wird diese allerdings seit Langem wegen ihrer interpersonellen Verteilungswirkungen und negativen Arbeits-

\footnotetext{
1 Vgl. z. B. Leiber, S./Zwiener, R. (2006): Eckpunkte zur Gesundheitsreform: Widersprüchlich und unzureichend, IMK-Report 13; Leiber, S. (2007): Nach der Reform ist vor der Reform: Ungelöste Fragen der Gesundheitsreform 2007, in: WSI-Mitteilungen 9, S. 508-510.

2 Beske, F./Baugärtner, M. (2008): Zu Lasten der Gesetzlichen Krankenversicherung. Auswirkungen politischer Entscheidungen auf die Finanzsituation der Gesetzlichen Krankenversicherung, Kiel.
}

Simone Leiber, Dr., ist Wissenschaftlerin im WSI in der Hans-Böckler-Stiftung. Arbeitsschwerpunkte: Wohlfahrtsstaaten im Vergleich, Europäische Sozialpolitik, Gesundheitspolitik.

e-mail: simone-leiber@boeckler.de 
anreize für verheirate Frauen im Hinblick auf eine sozialversicherungspflichtige Beschäftigung kritisiert. Der alleinige Schritt einer Steuerfinanzierung dieser versicherungsfremden Leistung zielt daher in die falsche Richtung. Denn damit würden die Gerechtigkeitsdefizite und frauenpolitisch negativen Implikationen verfestigt. Stattdessen sollte - gegebenenfalls mit Übergangslösungen für Menschen, die ihre Lebensentscheidungen bereits längerfristig und schwer reversibel auf das alte System zugeschnitten haben - über Möglichkeiten einer Einbeziehung von nicht-erwerbstätigen Eheleuten in die Beitragspflicht (z. B. auf der Basis von Mindestbeiträgen) nachgedacht werden. Die Kombination von beitragsfreier Mitversicherung und geringfügiger Beschäftigung, die das weibliche $\mathrm{Zu}$ verdienerinnen-Modell fördert, sollte abgeschafft werden.

Die Kosten versicherungsfremder Leistungen lassen sich im Rahmen des Fondmodells relativ einfach durch Steuern finanzieren. Dazu muss der Staat lediglich seine finanziellen Leistungen an den Fonds aufstocken. Rein technisch wäre eine stärkere Steuerfinanzierung auch ohne den Fonds möglich gewesen. Der Fonds macht die Zahlungsströme jedoch stärker transparent. Aus politischer Perspektive vermag er Finanzpolitiker dadurch möglicherweise eher zur Akzeptanz von Steuerzuschüssen zu bewegen als das alte, nur für wenige durchschaubare System des Risikostrukturausgleichs (RSA).

\section{INTEGRATION DER PRIVATEN KRANKENVERSICHERUNG}

Das (finanzielle) Potenzial, die Gesetzliche und die Private Krankenversicherung (PKV) zu integrieren, blieb bisher ungenutzt. Auch hier kann der Fonds als Hebel dienen. Aufgrund dessen, dass die Beiträge der Versicherten nun bereits von den Zahlungen an ihre Krankenkasse abgekoppelt sind, lassen sich die Mitglieder der PKV leichter in das einkommensabhängige Beitragssystem und Umverteilungssystem einbeziehen. Konkrete Modellvorschläge, unter anderem zur dann notwenigen Einbeziehung der PKV in den RSA, liegen bereits seit Längerem vor. ${ }^{3}$ Dies schafft mehr und fairen Wettbewerb zwischen PKV und GKV und verbessert die Einnahmen-Ausgabenrelation des Fonds, da die PKV relativ mehr Mitglieder mit höheren Einkommen als die GKV hat.

\section{WETTBEWERBSVERZERRUNGEN SCHNELLSTENS KORRIGIEREN}

Ein anderes Problem, das von verschiedensten Seiten kritisiert wird und das gravierende Wettbewerbsverzerrungen im neuen System hervorruft, sollte schnellstens behoben werden: So wirkt die im Rahmen des Gesundheitsfonds eingeführte individuelle Überforderungsklausel ${ }^{4}$ - obwohl sie aus sozialpolitischen Gesichtspunkten sinnvoll ist - zulasten von Krankenkassen mit vielen Einkommensschwachen. Kurzfristig wäre eine Abschaffung dieses Wettbewerbsnachteils durch Ausgleichszahlungen über den Fonds eine relativ einfache Lösung, die zu einer deutlichen Verbesserung der Rahmenbedingungen für den Wettbewerb um gute Versorgung (anstelle eines Wettbewerbs um Versicherte mit hohem Einkommen) führen würde. Längerfristig sollte man jedoch zur rein einkommensabhängigen Finanzierung zurückkehren. Dadurch würde dieses Problem obsolet - und die komplexe, Bürokratie erzeugende Regelung um die Zusatzbeiträge, einschließlich der Überforderungsklausel, gleich dazu.

\section{RÜCKKEHR ZU EINKOMMENS- ABHÄNGIGEN BEITRAGSSÄTZEN}

Schon heute ist absehbar, dass die Finanzierungskonstruktion um den Gesundheitsfonds dazu beitragen wird, die Finanzierungslasten des Gesundheitssystems weiter von den Arbeitgebern zu den Versicherten und von einkommensabhängigen zu einkommensunabhängigen Bestandteilen zu verschieben. Einkommensunabhängige Zusatzbeiträge belasten insbesondere Geringverdiener - und damit auch Frauen besonders stark. ${ }^{5}$

Die neu eingeführte Möglichkeit für die Kassen, im Rahmen des Fonds auch einkommensunabhängige Zusatzbeiträge zu erheben, wurde damit begründet, den Wettbewerb zwischen den Kassen zu intensivieren. Das Preissignal des Zusatzbeitrags - so das Argument - werde unmittelbarer wirksam, da es sich um einen konkreten Eurobetrag und nicht einen Beitrags-Prozentsatz handele. Zudem seien die Arbeitgeber nicht daran beteiligt, weswegen Beitragserhöhungen für die Versicherten stärker spürbar würden. Auf diese Weise erhoffte man sich, die Kassen zu mehr Effizienz und einem stärker diversifizierten Versorgungsangebot zu bewegen. Dass diese von den Zusatzbeiträgen erhofften Ef- fekte auch eintreten, ist jedoch zweifelhaft. Untersuchungen zum Kassenwechsel von Versicherten zeigen, dass die Wechselintensität in Deutschland auch zuvor bereits vergleichsweise hoch war und dass für eine Steigerung des Wechselpotenzials künftig weniger Preis- als Versorgungs- und Qualitätsoptionen ausschlaggebend sein werden. ${ }^{6}$ Gerde Letzteres wird durch das neue System jedoch infrage gestellt. In der ersten Phase nach dem Start des Gesundheitsfonds ist davon auszugehen, dass die Kassen unter allen Umständen versuchen werden, einen einkommensunabhängigen $\mathrm{Zu}$ satzbeitrag zu vermeiden. Dabei besteht die Gefahr, dass sie unter dem Sparzwang auch effiziente Leistungen und wichtige Investitionen in eine hohe Versorgungsqualität (z. B. im Bereich der integrierten Versorgung) beschneiden. Bei weiter steigenden Gesundheitskosten werden die Ausgaben längerfristig jedoch aus den Mitteln des Fonds nicht mehr zu decken sein, und die Kassen werden zu Zusatzbeiträgen greifen müssen. Wäre der Damm erst einmal gebrochen und müsste die Mehrheit der Kassen Zusatzbeiträge einführen, dürfte das Preissignal Zusatzbeitrag wieder an Bedeutung verlieren.

\section{ANREIZE FÜR QUALITÄTS- WETTBEWERB}

Die Herstellung eines Qualitätswettbewerbs sollte daher auf anderen Wegen gefördert werden. Dazu gehören etwa gezielte staatliche Anreize für Prävention und die integrierte Versorgung sowie weitere Flexi-

3 Sehlen, St./Hofmann, J./Reschke, P. (2006): Möglichkeiten zur Einbeziehung von gesetzlich und privat Krankenversicherten in eine integrierte Krankenversicherung, in: Albrecht, M. et al. (Hrsg.): Modelle einer integrierten Krankenversicherung, Berlin, S. 89-142.

4 Diese sieht vor, dass die im Rahmen des Fonds ermöglichten, allein von den Versicherten zu tragenden Zusatzbeiträge nicht mehr als $1 \%$ des beitragspflichtigen Einkommens der Versicherten ausmachen dürfen, soweit sie über $8 €$ liegen.

5 Manouguian, M./Greß, St./Walendzik, A./Wasem, J. (2008): Finanzielle Auswirkungen des Zusatzbeitrags auf die Mitglieder der gesetzlichen Krankenversicherung, Diskussionsbeitrag aus dem Fachbereich Wirtschaftswissenschaften, Universität Duisburg-Essen 166.

6 Vgl. u.a. Andersen, H. H./Grabka, M. M. (2006): Kassenwechsel in der GKV 1997-2004. Profile Trends - Perspektiven, in: Göpffahrt, D. et al. (2006): Jahrbuch Risikostrukturausgleich 2006: Zehn Jahre Kassenwechsel, Sankt Augustin, S.145-189. 
bilität in der Vertragsgestaltung der Kassen mit den Leistungserbringern. Ein zentraler Punkt ist ebenfalls die Weiterentwicklung des bisher auf 80 Krankheiten beschränkten morbiditätsorientierten RSA. Die Definition dieser Krankheiten war im Vorfeld der Reform höchst umstritten und muss weiter ausgebaut werden. Ein solcher Ausgleich ist das Herzstück eines jeden Systems, das auf dem Kassenwettbewerb beruht und keine risikobasierten Prämien kalkuliert. Nur auf diese Weise kann überhaupt die Voraussetzung dafür geschaffen werden, dass ein Wettbewerb um die Versorgungsqualität und nicht um Versicherte mit guten Risiken entsteht. Der morbiditätsorientierte RSA sollte auch nicht vorschnell allein dadurch in Verruf gebracht werden, dass das System prinzipiell durch Betrug missbraucht werden kann. Denn dies gilt für alle Bereiche, die auf der Dokumentation von Krankheitsbildern beruhen. Sie sind abhängig davon, ob Ärzte diese Dokumentation korrekt anwenden. Sollten sich die Anzeichen verdichten, dass es im Rahmen dieser neuen RSA tatsächlich zu Erpressungsversuchen der Kassen und bewusst fehlerhafter Dokumentation kommt, müssen Kontrollmechanismen eingeführt werden. Durch die Konzentra- tion der öffentlichen Diskussion auf Betrug darf jedoch nicht aus dem Blick geraten: dass der morbiditätsorientierte RSA überhaupt verwirklicht wurde, ist eine Errungenschaft. In Zukunft muss das Instrument verfeinert werden.

Die Anreize für einen Qualitätswettbewerb können und sollten daher auf anderen Wegen gesetzt werden, etwa durch die Weiterentwicklung des auf 80 Krankheiten beschränkten morbiditätsorientierten RSA, gezielte Anreize für Präventionsangebote und integrierte Versorgung sowie weitere Flexibilität in der Vertragsgestaltung der Kassen mit den Leistungserbringern.

\section{Wirtschafts- und Sozialwissenschaftliches Institut in der Hans-Böckler-Stiftung \\ in Kooperation mit \\ Deutscher Gewerkschaftsbund}

\section{Symposium „60 Jahre Tarifvertragsgesetz - Zukunft der Tarifautonomie"}

\section{Berlin, 23. und 24. April 2009}

Im April 2009 wird das Tarifvertragsgesetz 60 Jahre alt. Während dieses Gesetz seit seiner Verabschiedung in nahezu unveränderter Form die rechtliche Grundlage der praktischen Tarifpolitik in der Bundesrepublik Deutschland bildet, erfuhren die Tarifpolitik und die Tarifvertragsstrukturen in diesem Zeitraum einen tief greifenden Wandel.

Wir nehmen den Geburtstag zum Anlass für eine kritische Bilanz und einen Blick nach vorn. Was ist aus der Vergangenheit zu lernen und welche Perspektiven für eine erneuerte Tarifpolitik lassen sich entwickeln? Das Tarifpolitische Symposium von WSI und DGB will dazu ein Diskussionsforum bieten.

Mit wissenschaftlichen Beiträgen von Reinhard Bahnmüller, Reinhard Bispinck, Thomas Blanke, Thomas Dieterich, Heiner Dribbusch, Richard Hyman, Jürgen Kädtler, Thorsten Schulten, Sybille Stamm, Ulrich Zachert.

Mit gewerkschaftlichen Diskussionsbeiträgen von Margret Möning-Raane (ver.di), Werner Bischoff (IG BCE) und Michael Sommer (DGB).

sowie einer politischen Diskussionsrunde mit

Dieter Hundt (BDA), Bundesarbeitsminister Olaf Scholz, Ministerpräsident Peter Müller, Franz-Josef Möllenberg (NGG) und Berthold Huber (IG Metall).

Informationen zum Programm unter: http://www.boeckler.de/pdf/v_2009_04_23_programm.pdf Anmeldung im Internet unter: www.boeckler.de (Veranstaltungen) oder Katharina-Kruse@boeckler.de, Tel.: 0211/7778-111 